Recherches en didactique des langues et des cultures

Les cahiers de l'Acedle

7-1 | 2010

Notions en questions - Les plurilinguismes

\title{
Le projet Dylan ou les enjeux politiques, cognitifs et stratégiques du plurilinguisme
}

Anne-Claude Berthoud

\section{OpenEdition}

Journals

Édition électronique

URL : https://journals.openedition.org/rdlc/1981

DOI : $10.4000 /$ rdlc. 1981

ISSN : $1958-5772$

Éditeur

ACEDLE

Référence électronique

Anne-Claude Berthoud, «Le projet Dylan ou les enjeux politiques, cognitifs et stratégiques du plurilinguisme », Recherches en didactique des langues et des cultures [En ligne], 7-1 | 2010, mis en ligne le 01 avril 2010, consulté le 12 avril 2022. URL : http://journals.openedition.org/rdlc/1981 ; DOI https://doi.org/10.4000/rdlc. 1981

Ce document a été généré automatiquement le 12 avril 2022.

\section{c) (1) (9)}

Recherches en didactique des langues et des cultures is licensed under a Creative Commons AttributionNonCommercial-NoDerivatives 4.0 International License 


\title{
Le projet Dylan ou les enjeux politiques, cognitifs et stratégiques du plurilinguisme
}

\author{
Anne-Claude Berthoud
}

\section{NOTE DE L'AUTEUR}

Les trois premiers chapitres de ce texte sont issus de la partie scientifique et méthodologique du projet, élaborée conjointement avec G. Lüdi (Université de Bâle) et F. Grin (Université de Genève).

\section{Introduction}

1 Notre communication vise à présenter un aperçu du projet Dylan (Dynamique des Langues et Gestion de la Diversité), au sens où celui-ci interroge très précisément la thématique du colloque $\mathrm{NeQ}$ qui envisage le plurilinguisme dans toute sa diversité et son hétérogénéité :

- que ce soit au niveau des politiques linguistiques (institutionnelles, éducatives et économiques), au niveau des représentations des acteurs impliqués (représentations explicites ou implicites), ou encore au niveau des pratiques interactionnelles (professionnelles ou formatives) ;

- ou que ce soit au niveau des différents enjeux liés au plurilinguisme, en termes d'enjeux politiques, cognitifs et stratégiques.

2 Une diversité et une hétérogénéité qui seront néanmoins guidées par une conception spécifique du plurilinguisme, une conception plurilingue $\mathrm{du}$ plurilinguisme, envisageant le plurilinguisme non comme addition de plusieurs monolinguismes, mais sous la forme de répertoires plurilingues aptes à fonctionner comme ressources dans la diversité des contextes communicatifs. 
3 Dans un premier temps, nous présenterons les objectifs généraux du projet Dylan, ainsi que le cadre d'analyse. Puis nous esquisserons quelques premiers résultats de recherche, avant de passer à l'analyse d'un exemple de formation plurilingue à l'université, dans un cours de marketing.

\section{Les objectifs du projet}

Le projet Dylan poursuit trois objectifs interreliés.

4 Le premier objectif consiste à mieux comprendre comment les répertoires plurilingues se développent et sont mis en œuvre dans diverses situations de communication.

5 Le second objectif vise à identifier les conditions nécessaires pour que les répertoires plurilingues, qui sont une partie de l'héritage européen, contribuent activement au développement d'une société fondée sur la connaissance. Le projet part de l'hypothèse que deux types de bénéfices peuvent être tirés du plurilinguisme. Le premier est lié à l'efficacité de la communication dans des contextes plurilingues. Dans cette optique, le plurilinguisme peut être considéré comme un atout, non seulement au sens métaphorique du terme, mais au sens d'un véritable apport dans la construction et la transmission de l'information, dans la résolution de problèmes et le contrôle de l'interaction. Le second type de bénéfice est à saisir en termes de démocratie et de justice sociale, au sens où le plurilinguisme contribue à encourager des formes d'interaction sociale plus équitables et à la participation politique, contribuant par là à répondre au risque de déficit démocratique dû à l'élargissement de l'Union Européenne (UE).

6 Le troisième objectif du projet découle des deux premiers: doté d'une meilleure compréhension du plurilinguisme et des conditions dans lesquelles il peut devenir un avantage, le projet vise à formuler des recommandations politiques pour une gestion effective et démocratique de la diversité linguistique en Europe. Ces propositions doivent conduire à développer, au travers de mesures politiques, les conditions dans lesquelles le plurilinguisme européen peut se transformer en avantage individuel et collectif au sein d'une économie globalisée.

\section{Le cadre d'analyse}

7 Le cadre d'analyse vise à répondre à ces trois objectifs. Le développement et l'usage de répertoires plurilingues implique une approche qui soit pertinente aussi bien du point de vue de la recherche scientifique que du point de vue pratique des acteurs susceptibles de l'utiliser dans la sélection, la formulation, l'implémentation et l'évaluation de politiques concernant la diversité linguistique.

8 Cela implique que le cadre d'analyse puisse fournir une base conceptuelle tangible pour répondre aux enjeux épistémologiques et méthodologiques des différentes disciplines en présence. Il doit être par ailleurs suffisamment souple pour être à même de traiter des nouvelles questions qui émergent de la dynamique interne de processus de recherche orientés vers la pratique et répondre à l'exigence de saisir l'usage de ressources qui sont elles-mêmes structurées par leur usage dans des contextes spécifiques. Dans cette optique, il doit permettre de traiter les répertoires plurilingues aussi bien au niveau symbolique qu'au niveau discursif et interactionnel. Ces 
différentes exigences conduisent par conséquent à un cadre d'analyse constitué de dimensions dont il convient de saisir les interrelations.

\subsection{Dimensions et interrelations}

9 Quatre dimensions constituent les pièces conceptuelles maîtresses du projet :

- les pratiques langagières ;

- les représentations du plurilinguisme et de la diversité linguistique, observables au travers du discours et de l'interaction;

- les politiques linguistiques des états ou autres institutions d'état et institutions publiques (à l'échelle locale, régionale, nationale et supra-nationale), ainsi que les stratégies linguistiques des entreprises du secteur privé ;

- le contexte linguistique ou environnement linguistique dans lequel les acteurs opèrent.

10 Ces quatre dimensions sont interreliées de multiples façons pour saisir le développement et l'usage des répertoires plurilingues. Et il est à souligner qu'aucune de ces dimensions ou interrelations n'a a priori de préséance épistémologique ou méthodologique sur les autres.

Le cadre d'analyse peut être représenté de la façon suivante.

Figure 1 - Les quatre domaines et les douze flèches qui les relient.

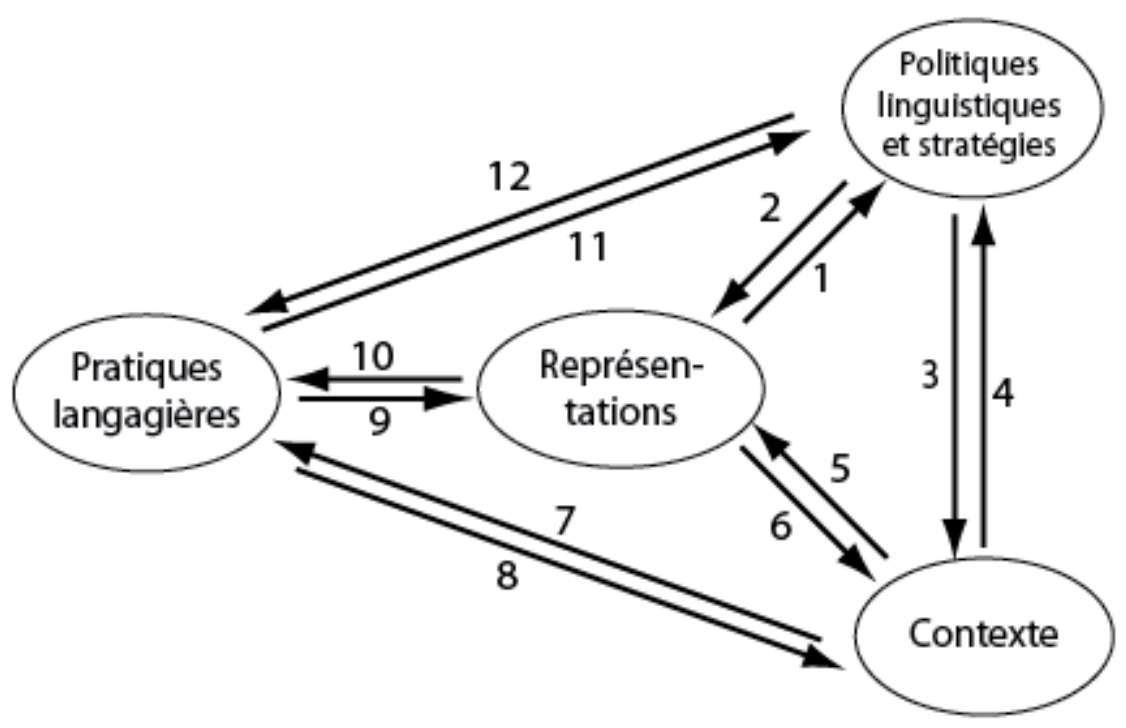

Chaque domaine de la figure 1 symbolise une relation susceptible d'être analysée dans l'examen des processus de développement et de mise en œuvre des répertoires plurilingues. Par exemple, la flèche 1 renvoie à la manière dont les représentations du plurilinguisme influencent les politiques et les stratégies linguistiques. La flèche 5 renvoie à la façon dont un contexte donné est perçu par les acteurs sociaux et influence leurs représentations du plurilinguisme. La flèche 4 montre comment la politique linguistique modifie le contexte et la flèche 7 , comment ces modifications ont un impact sur les pratiques langagières. Quant à la flèche 9 , elle réfère à la façon dont les pratiques langagières constituent des objets de représentations, exprimées dans le discours, et qui ont (flèche 1) un impact sur les politiques et les stratégies linguistiques. 


\subsection{Les terrains de recherche} terrains de recherche, ce terme étant pertinent pour l'organisation du travail empirique effectué au sein du projet Dylan. Trois terrains revêtent une importance particulière pour la gestion du plurilinguisme en Europe, à savoir, les entreprises du secteur privé, les institutions supranationales au sein des vingt-sept membres et les systèmes éducatifs.

13 Le choix des terrains, qui servent notamment à structurer les modules ou «workpackages » (WP) du projet, relève de critères conceptuels, méthodologiques et organisationnels.

14 Au plan conceptuel, ce choix se fonde sur la recherche actuelle portant sur l'interaction plurilingue, et qui inscrit le projet Dylan dans le paradigme d'une cognition située, où le contexte dans lequel l'interaction prend place est d'une importance capitale.

15 Au plan méthodologique, on admet que ces trois terrains-les entreprises, les institutions européennes et les systèmes éducatifs-constituent des situations opératoires et appropriées, aussi bien pour un développement conceptuel pertinent que pour les procédures de recueil des données dans les tâches de recherche. Au plan organisationnel, ce découpage facilite le travail de collaboration, ainsi que la communication et l'intercompréhension entre les équipes de recherche.

17 Plus informellement, ces trois terrains peuvent être vus comme des configurations naturelles de l'organisation pratique de la recherche.

18 Ce choix de terrains reflète par ailleurs l'hypothèse selon laquelle les modes de communication observés sur chacun des terrains peuvent avoir un caractère exemplaire, et mettre en lumière des modes de communication généralisables. S'il est évident qu'au sein de ces différents terrains, les processus mis en œuvre sont différents et que les questions de recherche qui en émergent ne sont pas les mêmes, il est possible néanmoins de faire l'hypothèse que les quatre dimensions présentées plus haut sont pertinentes pour les trois terrains. Leur mise en relation doit servir de point de référence pour le développement d'un plurilinguisme efficace et démocratique en Europe.

19 Les deux représentations bi-dimensionnelles du cadre d'analyse peuvent être ici étendues à trois dimensions, comme le montre la figure 2. 
Figure 2 - Le cadre d'analyse mis en perspective

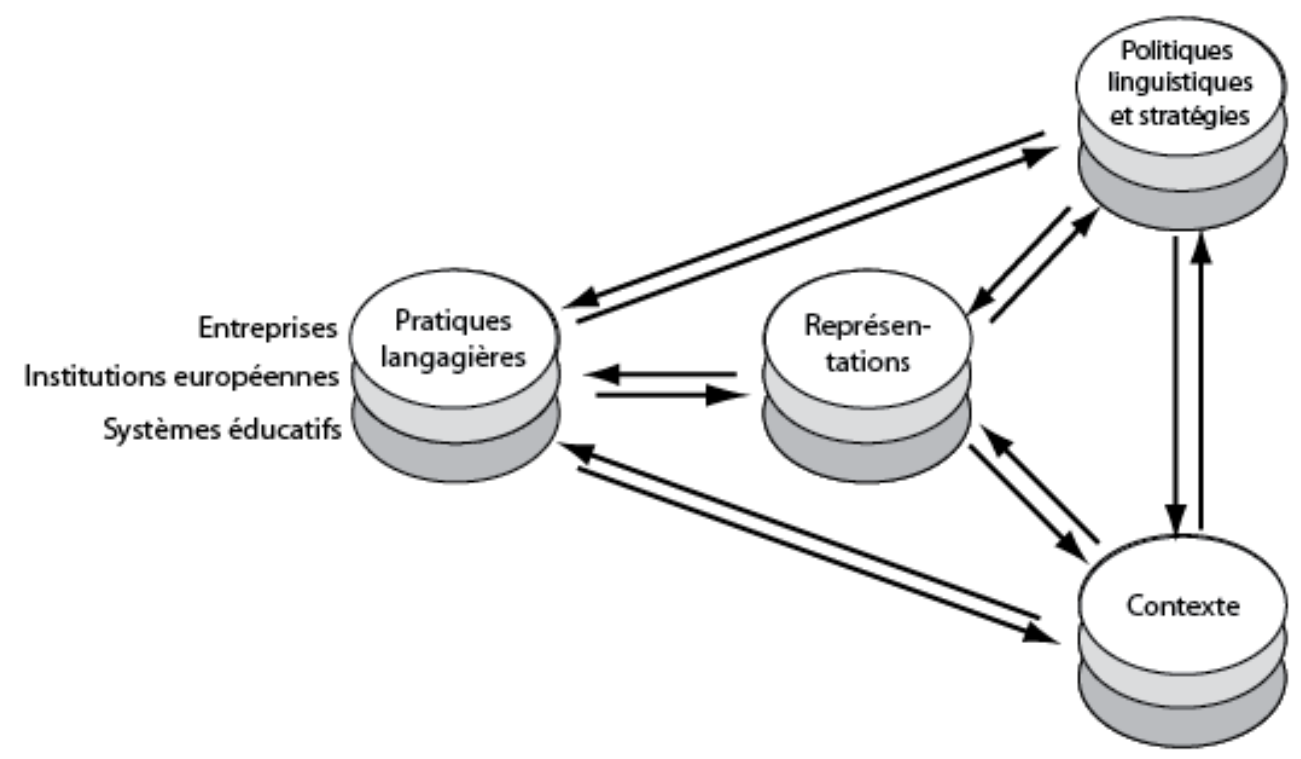

\subsection{Les questions transversales}

Les modèles qui envisagent le développement et la mise en œuvre des répertoires plurilingues doivent cependant tenir compte de trois types de facteurs supplémentaires, susceptibles d'être intégrés explicitement dans le cadre d'analyse.

La première question renvoie à l'une des idées majeures de Dylan qui consiste à donner des réponses appropriées aux défis posés par la diversité linguistique et à fournir la base de référence pour la mise en place de politiques linguistiques, aussi bien pour la gestion du plurilinguisme, aux niveaux local, régional, national et supranational, que pour l'identification de pratiques appropriées en situation d'interactions plurilingues.

2 Pour évaluer ce que la société peut faire pour structurer et orienter le plurilinguisme, et pour proposer des mesures pour une gestion efficiente et démocratique de la diversité linguistique, il s'avère essentiel de réexaminer les relations entre ces quatre dimensions, aussi bien en termes d'efficience (qui renvoie à la question d'une saine allocation des ressources) qu'en termes d'équité (qui réfère à la question d'une juste distribution des ressources).

3 La deuxième question renvoie à la langue en soi et constitue en quelque sorte la matière première du projet. Il s'agit ici plus particulièrement de mettre l'accent sur les formes et les règles qui émergent de l'interaction entre les locuteurs, et par là d'identifier et d'expliciter la façon dont les répertoires plurilingues sont, en tant que tels, des sources de changements linguistiques, ayant des conséquences importantes pour les fonctions de la communication et le statut social des langues en question.

La troisième question adopte une perspective historique. Les quatre dimensions présentées et leurs interrelations sont non seulement issues de l'ensemble complexe d'interactions discutées plus haut, mais elles sont aussi le produit d'une longue histoire ayant affaire avec la diversité linguistique et l'usage de répertoires plurilingues. On fera l'hypothèse que l'expérience historique de l'Europe avec le plurilinguisme influence de 
façon significative non seulement les représentations actuelles et les discours qui les manifestent, mais aussi les politiques; elle constitue par conséquent une partie importante de notre environnement linguistique. C'est dans ce sens qu'une perspective historique pour aborder les processus de développement et d'usage de répertoires plurilingues devrait informer le travail d'analyse effectué sur les interrelations entre les quatre dimensions.

Figure 3 - Le cadre d'analyse élargi

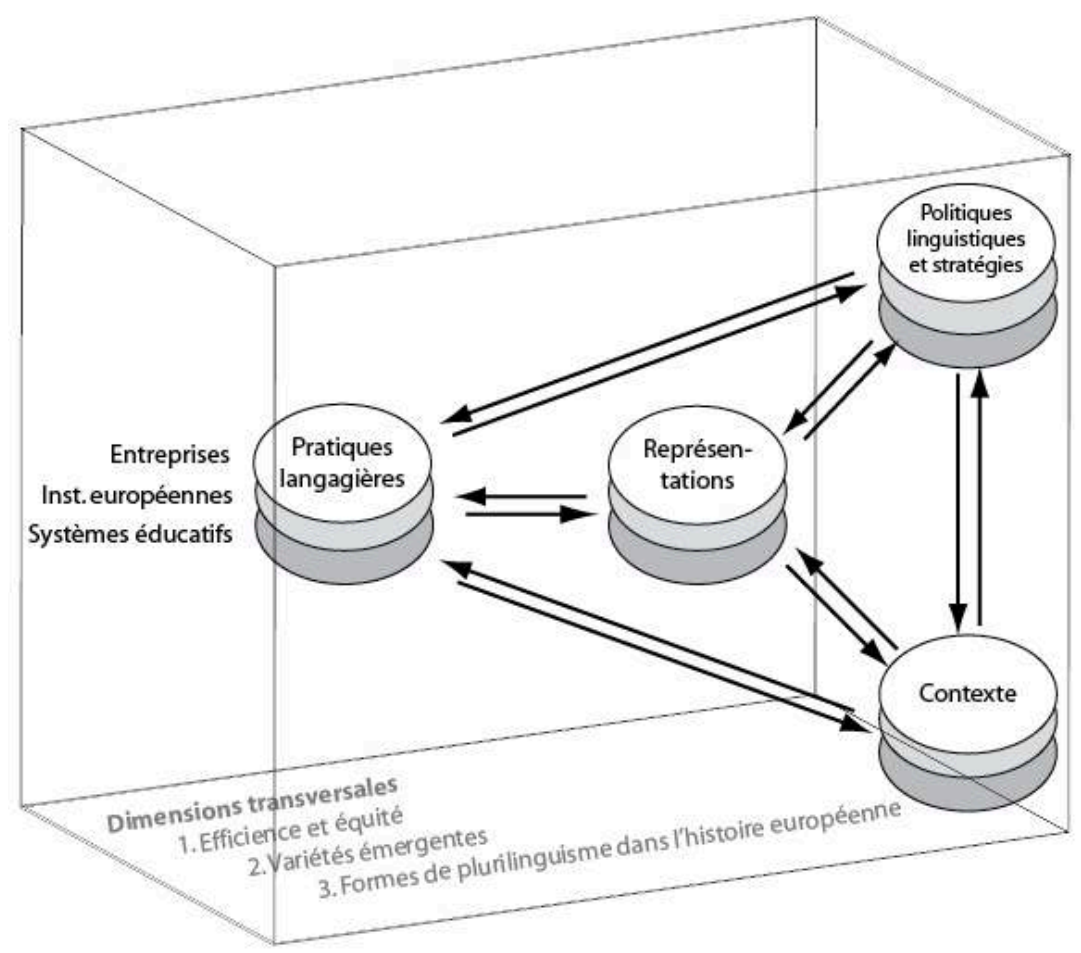

Ces trois types de questions - efficacité et justice sociale, variétés émergentes et formes de plurilinguisme dans l'histoire de l'Europe seront dès lors désignées comme des questions transversales.

\section{5. « Workpackages » et tâches de recherche}

Le travail effectué par les chercheurs dans le projet Dylan est organisé en quatre « workpackages » (WP) ou modules, avec deux WP complémentaires pour la formation et le management. Les trois premiers WP se concentrent sur les trois terrains décrits plus haut, chacun d'entre eux étant composé de trois à six tâches de recherches réalisées par équipes. Le quatrième WP est organisé de façon quelque peu différente et se compose des trois questions transversales - efficience et équité, variétés émergentes et formes de plurilinguisme dans l'histoire européenne - questions transversales prises en charge par trois équipes de recherche spécifiques qui interagissent avec les trois autres terrains. 


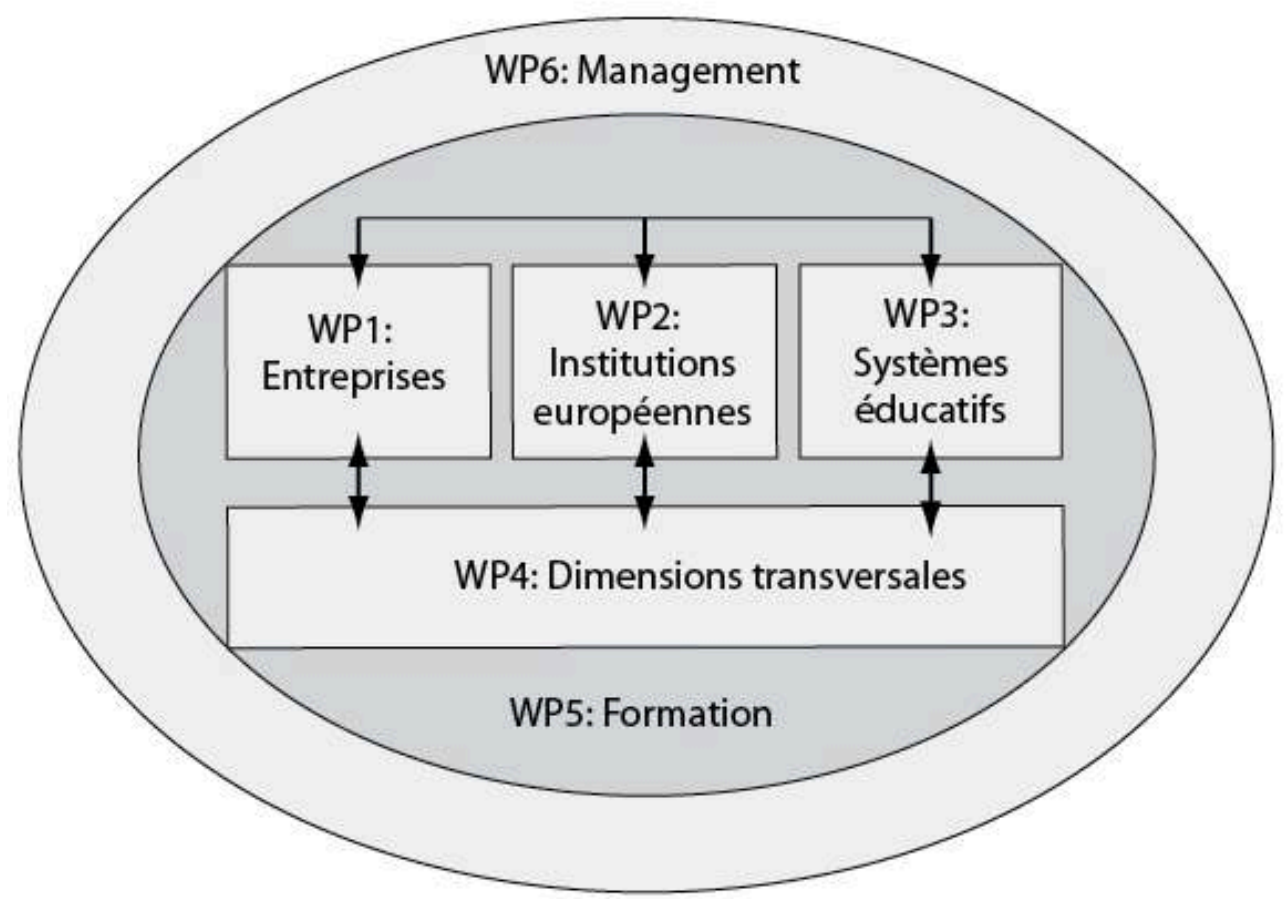

\subsection{1. « Workpackage » $1:$ les entreprises}

Ce WP a pour objet le monde du travail (des petites et moyennes entreprises aux organisations multinationales) au travers des quatre dimensions et leurs interrelations, les équipes de recherche se concentrant sur des dimensions et interrelations spécifiques.

Ce WP répond à un constat paradoxal : alors que les situations professionnelles sont de plus en plus marquées par une internationalisation des projets, des collaborations, des équipes, des partenariats, et alors même que ce phénomène apparaît avec une certaine évidence, les recherches qui étudient la manière dont les milieux professionnels font face à cette internationalisation dans leurs pratiques ordinaires, dans leur gestion des collaborations, dans leurs stratégies explicites ou implicites, dans leurs discours face au changement restent relativement peu nombreuses.

Le WP a pour objectif de répondre à ce manque, en procédant à des enquêtes de terrain qui montrent comment les professionnels issus de contextes très différents traitent pratiquement la question du plurilinguisme - que ce soit dans la manière dont ils organisent leurs réunions, structurent des pratiques de collaboration, prennent des décisions, se donnent des règles, négocient voire imposent un choix de langue, formulent des politiques et des prises de positions générales concernant l'emploi des langues dans l'entreprise.

Plus précisément, il s'agit de savoir dans quelle mesure les professionnels identifient les situations plurilingues caractérisant de nombreuses activités au travail comme un atout, comme une dimension qui signifie, dans le respect pour la diversité et dans l'expression de la diversité, une créativité accrue ; ainsi que de savoir, si tel n'est pas le 
cas, dans quelle mesure et sur la base de quelles expériences ils identifient plutôt le plurilinguisme à une dimension problématique, porteuse de difficultés et d'obstacles.

31 Le WP est lui-même emblématique de cette diversité, puisqu'il réunit des équipes ayant des références théoriques, des focus analytiques et des compétences méthodologiques complémentaires, permettant d'envisager et de traiter des aspects très divers, par des approches diversifiées, du plurilinguisme au travail.

\subsection{2. « Workpackage » 2 : les institutions européennes}

32 Ce WP étudie les relations entre pratiques langagières, représentations et politiques linguistiques dans le contexte des institutions européennes (la Commission Européenne, le Parlement et le Conseil de l'Union Européenne), avec une focalisation sur des dimensions et interrelations spécifiques selon les partenaires.

De façon générale, ce WP vise à saisir comment les institutions européennes envisagent la question de la communication interne et de la communication externe. Il cherche à décrire les conditions et motivations des différents choix de langues au sein des institutions européennes, ainsi qu'à comprendre les motivations au niveau micro des individus et au niveau macro des idéologies qui configurent la communication plurilingue à l'intérieur et à l'extérieur des institutions.

Ce WP porte une attention particulière à la façon dont les changements rapides du contexte modifie les politiques et les stratégies linguistiques dans les institutions, et comment ces changements se reflètent dans les règlements et recommandations. Les politiques et les stratégies linguistiques se trouvent confrontées à de nouvelles réalités économiques qui font émerger de nouvelles pratiques.

Il cherche en particulier à montrer comment les pratiques plurilingues au sein des institutions servent de modèles et ont un impact sur les pratiques sociales et la définition du contexte. Dans cette perspective, il aborde notamment la question du risque d'un monolinguisme interne grandissant au sein des institutions européennes (communication interne) sur l'environnement social et la démocratie (communication externe).

En se fondant sur des documents politiques et la façon dont ces documents émergent des discussions à plusieurs niveaux hiérarchiques, ce WP analyse par ailleurs comment les institutions européennes envisagent le plurilinguisme et leur marge d'action possible pour l'influencer. Il s'agit de comprendre le rôle du plurilinguisme dans la définition de l'image des institutions elles-mêmes, en tant que label et valeur ajoutée.

Ce WP envisage également la question des représentations et de leur influence sur les pratiques langagières, sur les choix de langues et leur fonction comme normes internes.

\subsection{3. « Workpackage » $3:$ les systèmes éducatifs}

Ce WP vise à étudier les relations entre pratiques langagières, politiques linguistiques et représentations dans le contexte des systèmes éducatifs, et en particulier de l'enseignement tertiaire, avec une focalisation sur des dimensions et interrelations spécifiques selon les équipes de recherche.

Ce WP aborde notamment la façon dont les changements rapides du contexte (au sens large du terme) peuvent modifier les politiques et stratégies linguistiques mises en 
place dans les institutions éducatives et il cherche à saisir comment ces changements se manifestent dans la législation et les règlements de ces institutions. Les politiques et stratégies linguistiques se trouvent confrontées à de nouvelles réalités socioéconomiques qui déterminent de nouvelles pratiques langagières. Celles-ci génèrent de nouveaux discours sur le plurilinguisme, de nouvelles représentations, de nouvelles demandes pour la formation au plurilinguisme (et en particulier une formation Clil ou Emile $^{1}$ : enseignement intégrant langue et discipline), de nouvelles politiques linguistiques, ainsi que de nouveaux standards éducatifs.

Du point de vue des pratiques éducatives, et en particulier de Clil, le WP s'attache en particulier à décrire les effets cognitifs, argumentatifs et stratégiques des pratiques plurilingues, tant au niveau de l'enseignement qu'au niveau de la recherche.

41 Ces nouvelles pratiques (entendues au sens d'apprentissage et au sens d'usage) sont également confrontées à des modes d'apprentissage traditionnels monolingues. Ce WP examine par ailleurs l'impact des politiques linguistiques (aux niveaux étatique, régional et local) sur les systèmes éducatifs, ainsi que les effets en retour des nouvelles pratiques sur les stratégies et politiques linguistiques. Il examine également les inefficacités possibles des mesures proposées, les zones de divergence entre stratégies explicites et stratégies implicites, les décalages entre les représentations et les pratiques effectives, et vise ainsi à évaluer dans quelle mesure ces résultats peuvent informer de nouvelles recommandations et actions politiques.

\subsection{4. « Workpackage » 4 : les questions transversales}

43 Ce WP présenté au point 2.3, interagit de façon transversale avec les trois autres worpackages fondés sur les terrains des entreprises, des institutions européennes et des systèmes éducatifs. La fonction de ce « workpackage » est d'assurer une intégration des visées théoriques et méthodologiques des questions envisagées dans les trois autres WP. 


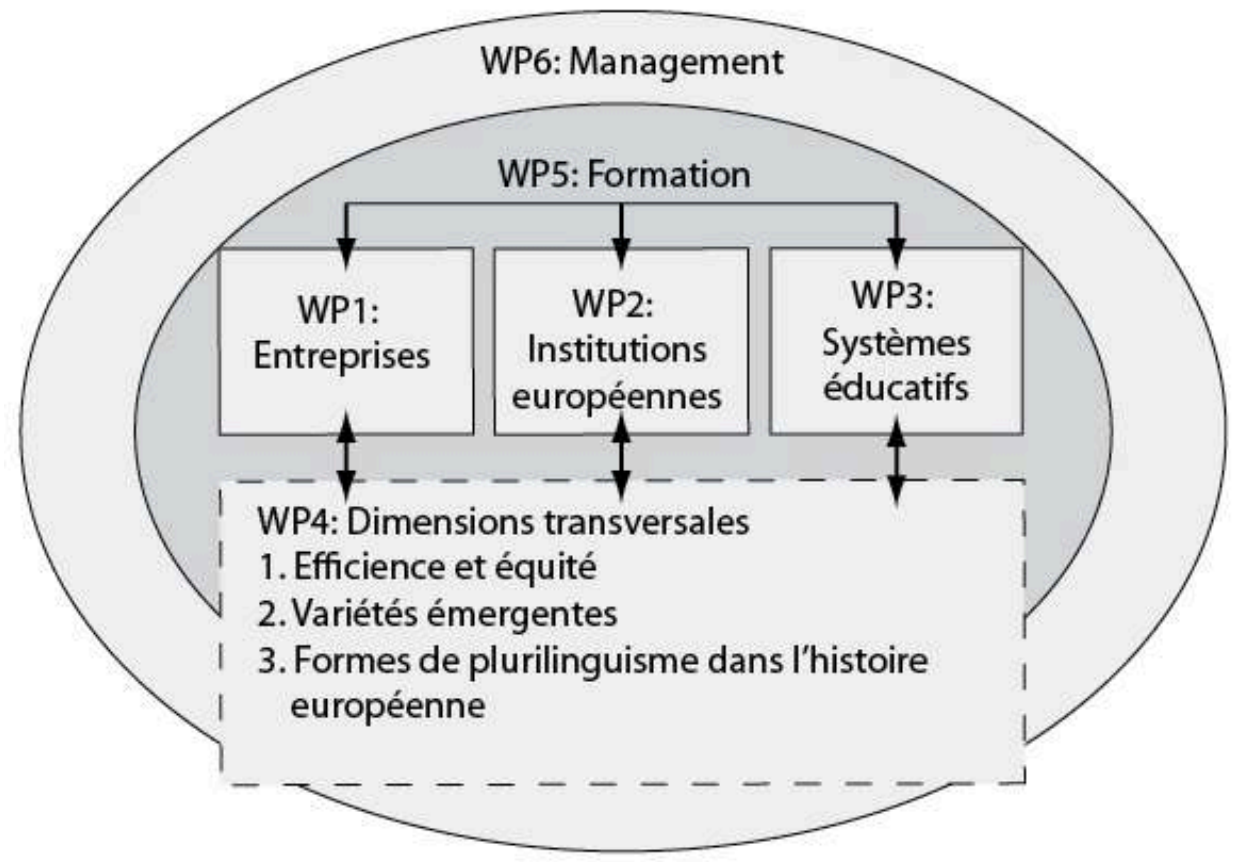

\section{Premiers résultats d'analyse}

Après deux ans de recherche, un certain nombre de " résultats " généraux peuvent être dégagés des "working papers" et des nombreuses séances, ateliers thématiques et méthodologiques organisés dans le cadre des séances du Consortium et dans le cadre d'ateliers spécifiques organisés par les WP.

Ces résultats sont à saisir essentiellement en termes conceptuels et méthodologiques.

\subsection{Impact cognitif et stratégique du multilinguisme}

Du point de vue de la question générale concernant l'impact et les conditions de l'impact des répertoires plurilingues sur la construction, la transmission et la mise en œuvre des connaissances (effets cognitifs et stratégiques), et en particulier dans le cadre de l'enseignement plurilingue universitaire, émergent déjà des résultats intéressants, en termes de profondeur et de richesse conceptuelle, de la mise en réseaux originale des concepts, de nouveaux moyens de conceptualisation, de développement des processus métalinguistiques, de problématisation plus explicite des savoirs transmis et construits, tout en prenant la complexité linguistique comme condition d'un meilleur accès aux savoirs.

Des résultats pertinents se font jour également en termes d'impact des répertoires plurilingues sur les processus interactionnels, tels que l'organisation séquentielle des interactions, sur les tours de parole, sur l'organisation des cadres participatifs, et en particulier le développement du leadership, ainsi que sur les changements de topics, ou encore d'impact des répertoires plurilingues sur l'organisation plus générale des 
activités, de contrôle de l'action et de l'interaction, de la résolution de problèmes ou de la prise de décision.

\subsection{Rôle du contexte sur l'intégration langue / connaissances} linguistiques apparaissent sous une nouvelle perspective au sein des études empiriques. Les représentations du plurilinguisme, comme les discours prescriptifs, sont euxmêmes enchâssés et ancrés dans les pratiques administratives, bureaucratiques et institutionnelles et les représentations du plurilinguisme ne sont pas autonomes, mais en prise directe sur les pratiques des différents acteurs sociaux. Ainsi, des bribes de discours prescriptifs émergent-elles des pratiques elles-mêmes sous la forme d'énoncés métalinguistiques.

\subsection{Une nouvelle définition du contexte}

Des études de cas et analyses empiriques ont produit de nouvelles discussions conceptuelles au sein des «WP». Elles concernent en particulier la définition et la catégorisation de la diversité linguistique et les articulations entre représentations, 
politiques et pratiques. La diversité des situations plurilingues a été mise en évidence : les dynamiques plurilingues sont fortement sensibles au contexte - chaque contexte configure des pratiques plurilingues spécifiques. Ainsi, le plurilinguisme apparaît-il sensible aux réalités locales tout en étant orienté en fonction d'enjeux globaux, localement pertinents.

\subsection{Des politiques et stratégies à la gestion linguistique}

53 La question de l'impact des politiques et des stratégies linguistiques implique une redéfinition notoire de ces notions qui s'avèrent insuffisamment opératoires pour rendre compte de la complexité des processus impliqués. Ce qui conduit à les remplacer par la notion de "measures of language management " (gestion linguistique), désignant toutes formes d'intervention de l'entreprise ou de l'institution sur les répertoires de leurs employés (p. ex. normes quant à l'usage des langues, cours de langue, politique d'embauche) - qu'elles soient explicitement formulées ou non, à condition d'être observables dans la pratique.

\subsection{Relation indirecte entre politiques et pratiques} université, d'une faculté / d'un département. Pour mesurer les résultats, il convient de rechercher les traces de l'implémentation de ces politiques / normes - et elles sont différentes à chaque niveau.

Observer les traces de l'implémentation de ces politiques implique que l'on fasse la distinction entre «outputs» et "outcomes». Il s'agit de saisir les facteurs qui déterminent les politiques (politiques $=$ variables dépendantes), sur leurs résultats (output) et conséquences (outcomes), et dès lors de distinguer d'une part des politiques ouvertement déclarées (mesures législatives, normes formulées par les systèmes éducatifs et les universités) et cachées (qu'est-ce qui se fait réellement dans la pratique selon les observations des chercheurs ou selon les informations recueillies lors d'entretiens). 


\subsection{Recatégorisation des représentations} pratiques sont plus riches et complexes qu'elles n'étaient posées au départ et que les catégories qui permettent de les appréhender sont elles aussi à reconsidérer. Si l'hypothèse voulait que les interviews appellent plutôt des représentations partagées et préconstruites et que les pratiques laissent émerger des représentations individuelles, on observe ici que des représentations individuelles peuvent émerger des discours (dans les interviews) - représentations non situées - et que des représentations partagées peuvent émerger des pratiques discursives elles-mêmes - représentations situées. Cela conduit à une autre façon d'aborder les représentations, c'est à dire d'adopter un point de vue émique, visant à accéder à la façon dont les acteurs euxmêmes catégorisent les représentations, et notamment dans les déclarations métalinguistiques qui émergent dans l'interaction.

Ces déclarations métalinguistiques apparaissent comme des traces privilégiées de la tension et de la dynamique qui s'instaurent entre les différents types de représentations, qui sont désormais à analyser dans leur continuité et leur intrication.

\subsection{En bref}

61 L'accès aux rationalités qui émergent des pratiques constituent à notre sens une source essentielle pour informer les futures politiques, pour que celles-ci puissent effectivement avoir "prise» sur les pratiques, pour qu'elles fassent sens pour les pratiques et qu'elles ne soient pas de simples déclarations/discours à valeur symbolique, sans conséquences pratiques.

Un autre type de partenariat entre politique et pratique se pose ainsi comme première condition de l'atout plurilingue.

\section{Un exemple à l'appui de ces résultats}

\section{De l'impact des stratégies linguistiques sur les pratiques dans
d'enseignement supérieur (stratification, " outputs " and " outcomes»).} Exemple de l'Université de Lausanne (Unil)

Pour montrer tout à la fois les relations indirectes entre politiques et pratiques, la nécessité d'introduire une stratification entre les différents niveaux impliqués, ainsi que l'importance de distinguer entre politiques explicites et politiques implicites, nous prendrons ici un exemple issu de la tâche de recherche lausannoise, tiré du WP 3 de Dylan, qui a pour objectif l'analyse des effets du plurilinguisme sur les processus de construction, de transmission et de mise en œuvre des connaissance (effets cognitifs et stratégiques), la confrontation de ces pratiques plurilingues aux représentations des différents acteurs impliqués, ainsi que la mise en rapport de ces pratiques et représentations avec les stratégies linguistiques mises en place par les institutions d'enseignement supérieur.

L'exemple analysé ici est tiré d'un cours de marketing dispensé à la Faculté des Hautes Études Commerciales (HEC) de l'Université de Lausanne. 


\subsection{Stratégies linguistiques de l'UNIL}

L'Université de Lausanne a récemment édicté des règles précises concernant les langues d'enseignement à tous les niveaux de cursus :

La langue officielle de l'Université est le français. Des enseignements peuvent être donnés dans d'autres langues. Mais demeurent réservés les enseignements prodigués et les examens assurés par les sections de langues de la Faculté des lettres et les enseignements de langues des autres facultés, autres que le français. Des enseignements peuvent être donnés dans d'autres langues que le français :

- pour autant que ces enseignements soient obligatoires et qu'aucune option de remplacement ne soit proposée en français, et que les étudiants aient la possibilité de bénéficier au préalable d'une formation linguistique appropriée et correspondant à leurs besoins spécifiques ;

- pour autant que l'enseignant maîtrise la langue d'enseignement et d'examen - le français et l'autre langue d'enseignement - au minimum au niveau B2/C1 (selon le Cadre européen commun de référence pour les langues du Conseil de l'Europe) et qu'il porte une attention particulière à la relation entre le contenu disciplinaire et son expression linguistique appropriée ;

- pour autant que les enseignements dans d'autres langues, les descriptions de programmes et modules, comportent des indications précises sur les langues utilisées et le seuil minimal de compétences linguistiques requis, dans les termes du Cadre européen commun de référence pour les langues, afin d'assurer une compréhension commune et internationale.

Au niveau du Bachelor

Les enseignements de la première année du Bachelor sont nécessairement en français.

Dès la $2^{\mathrm{e}}$ année, si la langue d'enseignement est en principe le français, des cours peuvent exceptionnellement être donnés dans une autre langue, pour autant que le plan d'études de l'année précédente ou des années précédentes prévoie que l'étudiant puisse acquérir les compétences linguistiques nécessaires et adaptées au niveau requis.

Les examens peuvent être présentés, au choix de l'étudiant, soit en français soit dans la langue du cours (sous réserve des cas mentionnés à l'article 2).

Au niveau du Master

Les facultés ont le choix de la langue d'enseignement et d'examen et s'engagent à informer clairement les étudiants à ce sujet.

Cependant, dans la perspective de l'encouragement du plurilinguisme, il est recommandé que tout programme inclue plusieurs langues d'enseignement ou des « modules de langues » dont la langue est différente de la langue d'enseignement.

67 Chaque faculté a été conduite à élaborer une politique des langues d'enseignement en fonction de ces directives générales, dont voici l'exemple de la faculté des Hautes Études Commerciales.

\subsection{Stratégies linguistiques de la Faculté des Hautes Études Commerciales}

\section{Utilisation des langues :}

- Examens - L'énoncé de l'examen est rédigé dans la langue du cours. L'étudiant peut, à tous les niveaux de formation, rédiger ses examens en français, quelle que soit la langue du cours.

- Mémoires - La rédaction des mémoires se fait en français ou en anglais, selon entente entre enseignant et étudiant. L'allemand ne peut pas être exigé, mais l'enseignant peut l'accepter.

\section{Langue d'enseignement :}

- Au niveau du Bachelor, conformément aux directives, l'enseignement en 1e et 2e année (cours obligatoires) se déroule en français, au moins pour l'une de deux sections lorsqu'il y a dédoublement. L'enseignement en 3e année de Bachelor (cours optionnels) se déroule soit en français, soit en anglais. Quelques enseignements en allemand sont envisagés.

- Au niveau des masters, l'enseignement se déroule soit en français soit en anglais. Certains masters sont entièrement en anglais (Finance, Économie politique), d'autres le sont en grande partie (Management). 
- Au niveau doctoral, l'enseignement se déroule généralement en anglais.

- À tous les niveaux, dans le cas de cours dispensés en français, une partie du matériel pédagogique peut être en anglais.

\section{Enseignants non francophones :}

- Les enseignants non francophones recrutés sur des postes de PO ont l'obligation d'enseigner en français après une période d'adaptation (entre 1 et 3 ans). Le décanat s'efforce de faire respecter cette règle, mais il devient progressivement plus difficile d'en assurer le respect, le recrutement des professeurs se faisant de plus en plus au niveau international, de façon prépondérante sur la base de la qualité des publications. La contrainte d'enseigner en français ne s'applique pas aux professeurs assistants.

Le décanat encourage tous les professeurs non francophones à suivre des cours de français (sans toutefois en faire une obligation).

\subsection{Stratification, « outputs » and « outcomes »}

68 facultés (exigences linguistiques requises pour les étudiants et les enseignants, programmes plurilingues, information des étudiants, etc.), ce sont les conséquences réelles / outcomes qui nous intéressent plus particulièrement. En partant des offres (« outputs») disponibles, on se demandera dans quelle mesure les acteurs en profitent, comment elles fonctionnent, quelle est leur qualité, etc. en employant des méthodes qualitatives (analyse de discours, analyse conversationnelle, etc.), mais aussi quantitatives (nombre de cours offerts dans d'autres langues, nombre d'étudiants inscrits dans des cours, etc.).

71 Par ailleurs, parmi les masters indiqués comme plurilingues dans le document de la faculté des HEC, certains présentent néanmoins des pratiques exclusivement monolingues (en anglais), montrant un décalage évident entre politiques linguistiques déclarées de la faculté et politiques implicites, en terme de conséquences effectives de ces politiques déclarées dans les pratiques mises en œuvre. D'où l'importance de considérer chacune des « strates » de la stratification évoquée plus haut.

\subsection{Stratégies linguistiques et représentations individuelles}

De plus, il apparaît qu'au niveau individuel des enseignants, il convient également de considérer un autre niveau de politique déclarée - celle issue des entretiens qui ont été conduits sur le « terrain » de HEC - et notamment celle émise par l'un des enseignants 
qui, conscient des hiatus qui apparaissent entre les différentes strates de politique linguistique, d'une part, et entre politiques et pratiques effectives, d'autre part, s'est construit ses propres stratégies linguistiques, qu'il a développées dans un ouvrage de référence. Il y développe en particulier la question de l'influence des différentes langues sur les stratégies et politiques dans son domaine et a notamment publié une série d'ouvrages de référence qui intègrent la dimension comparative. De plus, il dirige une thèse portant sur l'influence de la langue chinoise sur les modes de management : la hiérarchie, etc., et sur certains aspects du marketing. Il relate en particulier un exemple qui l'a conduit à la prise de conscience de l'existence d'une différence au niveau conceptuel résultant du facteur langue, le vocabulaire n'étant pas homogène et les traductions n'étant que partiellement possibles. Il donne l'exemple d'un concept de comptabilité qu'il présentait en français dans un cours d'introduction et dont il croyait savoir ce qu'il signifiait jusqu'au jour où un étudiant germanophone a mis en cause son explication, se basant sur la traduction de ce concept en allemand. Il a réalisé que l'interprétation du concept divergeait passablement selon la langue dans laquelle le concept avait été traduit. De manière générale, les langues exercent, selon lui, une influence sur les disciplines et en particulier sur sa discipline qui est le marketing. Son regard se situe à un niveau spécifique, dans la mesure où il traite moins du plurilinguisme comme objet de recommandation que du plurilinguisme comme moyen de recommandation. Il affirme aussi que dans ses cours en anglais L2, le travail sur le marketing l'incite à souligner davantage la question de la diversité des langues et des cultures et il qualifie le plurilinguisme de "ressource inexploitée», tout particulièrement dans le contexte institutionnel dans lequel il travaille, où le savoir sur les différences conceptuelles n'est considéré que comme soft skill, et donc non central. Tout en nous révélant cette prise de conscience de l'impact des langues et des cultures sur les savoirs, il identifie en même temps les limites de cet impact, tout spécialement pour son domaine, le management. Il considère que le management est relativement neutre par rapport à la langue comparé par exemple au droit ou à la comptabilité ${ }^{2}$. Ceci serait dû, selon lui, à son caractère monolingue et monoculturel. Les savoirs et savoirfaire ont été développés essentiellement aux États-Unis tandis que dans les autres langues, il s'agit uniquement d'une adaptation qui ne changerait pas le fond de la discipline. Les langues seraient un enjeu pour la mise en œuvre de celle-ci, mais pas pour ses concepts. De plus, il déclare ne pas devoir recourir à une réflexion spécifique pour pouvoir présenter sa discipline dans l'une ou l'autre langue, car ses propres pratiques d'enseignements ont toujours été parfaitement bilingues, dans la mesure où il a toujours enseigné cette matière en anglais et en français. Une "théorie » certes pleine de contradictions... mais qui a néanmoins le mérite de thématiser la question des langues pour les disciplines.

\subsection{Vers une " standardisation épaisse »}

73 Ce qui nous paraît plus intéressant encore, c'est la façon de thématiser le paradoxe issu de la double exigence de devoir aujourd'hui répondre au défi de la globalisation (par l'anglais) et de diversification (par le plurilinguisme) et sa tentative d'y répondre au travers d'une solution qu'il intitule "standardisation épaisse", qui serait une façon possible d'articuler unité et diversité. Pour lui, il ne s'agit pas de faire l'éloge de la différence radicale et que l'on nie les avantages de la standardisation, le risque étant de tomber dans une défense « rétro » et passéiste de la diversité, et souvent perçue comme 
de la pure nostalgie. Il s'agit bien plutôt de montrer la dynamique complexe entre diversité et standardisation et de concevoir le différent dans le semblable, la diversité dans l'unicité. Le rapport au standard et la volonté d'arriver à un seuil minimal d'intercompréhension doit se faire d'abord dans l'ordre de la détection de la standardisation trompeuse. Ainsi, l'anglais comme langue unique conduit à la fausse impression de partage du sens. Néanmoins, plutôt qu'une négation du standard, qui tend de toute façon à s'imposer, dans un monde globalisé, il convient de chercher une nouvelle forme de standardisation, une "standardisation épaisse » (expression dérivée du concept de «thick description» de l'anthropologue C. Geertz), une standardisation, qui, ajouterons-nous, contienne les traces d'une confrontation plurilingue. La «standardisation épaisse » consiste plus précisément à examiner un objet dans un contexte plurilingue afin d'augmenter son identification commune (le sens réellement partagé ou non partagé par des locuteurs de différentes langues), de détecter les configurations de sens produites par une langue particulière et leur influence sur différents aspects $d u$ processus de recherche. On peut ainsi tirer de cette "standardisation épaisse » une meilleure compréhension des objets et des phénomènes dans lesquels ils s'insèrent, à la fois, parce que la description est plus riche de sens et que les aspects partagés et non partagés de ce sens sont clairement énoncés et compris.

\subsection{Un exemple de pratique d'enseignement}

Enfin, tentons de voir comment ces "déclarations » issues des entretiens se trouvent effectivement mises en pratique dans son enseignement.

L'exemple que nous prendrons relève d'un enseignement dispensé en anglais L2 de l'enseignant et des étudiants. Il s'agit d'un cours magistral qui s'adresse à un public d'environ 80 étudiants de la faculté des Hautes Études Commerciales (HEC) qui sont inscrits en Maîtrise universitaire ès Sciences en management (Master of Science in Management). Cet enseignement a pour intitulé «International Marketing». Cette formation dure deux ans et est bilingue dans la mesure où les enseignements obligatoires se déroulent en anglais et les enseignements électifs, partiellement en anglais et partiellement en français. L'objectif de ce cours est «de familiariser les étudiant-e-s avec les principales situations et les principaux choix d'implantation internationale et de politique de marketing auxquels font face les entreprises opérant dans l'environnement global et d'apporter les connaissances de base concernant l'influence de la culture et de la langue sur différents aspects du marketing et de développer des éclairages précis sur des thèmes sélectionnés de marketing management international ». L'objectif mentionné annonce une particularité du marketing international, tel que l'enseignant l'interprète, et qui réside dans le fait que la diversité linguistique et culturelle fait partie de son objet d'étude. Il s'agit notamment de la séquence «sweet »:

\begin{tabular}{|l|l|l|}
\hline TdP & Loc & Séquence "Sweet" \\
\hline 1 & US & local taste/ . for similar items: \ ok \\
\hline 2 & & $\ldots$ \\
\hline 3 & US & we all know that products can be: . made with: . a little a content of sugar/ \\
\hline
\end{tabular}




\begin{tabular}{|c|c|c|}
\hline 5 & & .. \\
\hline 6 & US & $\begin{array}{l}\text { maybe I (already) explained this about süß:/ .. ((prononciation française)) <versus> } \\
\text { sucré }\end{array}$ \\
\hline 7 & US & did I:/ \\
\hline 8 & & $\ldots$ \\
\hline 9 & US & no/ \\
\hline 10 & & $(1.1)$ \\
\hline 11 & US & or you do not remember/ \\
\hline 12 & & $(0.8)$ \\
\hline 13 & US & or I really did not do it/ \\
\hline 14 & & .. \\
\hline 15 & US & $(($ rapide et bas $))<$ ok> \\
\hline 16 & & $(0.8)$ \\
\hline 17 & US & $\begin{array}{l}\text { so ((rire)) let me/ .. if-\&if you take/ .. what are/ pure . lexical equivalents } \backslash \text {. more or } \\
\text { less } \backslash(1.2) \\
\text { sucré:/ sü:ß/ (2.0) and sweet } \backslash \text {. ((plus bas }))<0 k \backslash>\end{array}$ \\
\hline 18 & & $(2.1)$ \\
\hline 19 & US & $\begin{array}{l}\text { so there is a POsitive .. value judgement . ON .. sweet/ . and süß\\
&in german/ . and in } \\
\text { english you have } \\
\text { a positive value judgement/ aspiration?)) which is indicated by the FIGUrative sense }\end{array}$ \\
\hline 20 & US & $\begin{array}{l}\text { ((bas et rapide) })<\text { ok }>\& y o u \text { have the proper sense/ . and you have the figurative- } \\
\text { \&figurative is/ ((aspiration)) when you say } \\
\text { for instance/.((plus lent) })<\text { my sweet darling }>\backslash \text {. ((baisse de volume }))<0 k \mid>\end{array}$ \\
\hline 21 & & $\ldots$ \\
\hline 22 & US & $\begin{array}{l}\text { and I say it in french/ . you-\&you can not say/ . ((plus lent)) <mon chéri sucré> \(1.0) ou } \\
\text { ma ((pronon. de la terminaison } \\
\text { fém. })) \text { s<chérie: }>\backslash((\text { aspiration?)) you can say/ . ((plus lent)) <ma DOUC :E . chérie> } \backslash \\
((\text { baisse de voix) })<\text { for instance }>\end{array}$ \\
\hline 23 & & $\ldots$ \\
\hline 24 & US & looks a little bit nineteenth century $\backslash$ \\
\hline 25 & ETs & ((rires des étudiants)) \\
\hline
\end{tabular}




\begin{tabular}{|c|c|c|}
\hline 26 & US & borderline $\backslash \mathrm{ok} /$ \\
\hline 27 & US & $\begin{array}{l}\text { (aspiration)) but typically/ (1.4) you have a taste/ (0.9) ok/ .. you have two sort of taste } \\
\text { (so) very basic/ }\end{array}$ \\
\hline 28 & US & (1.0) ((écrit au tableau)) \\
\hline 29 & US & you have salty/ (1.3) and you have sweet $\backslash$ \\
\hline 30 & US & (0.5) ((écrit au tableau)) \\
\hline 31 & US & ok $\backslash$ \\
\hline 32 & US & (0.5) ((écrit au tableau)) \\
\hline 33 & US & and in in many countries/ (0.8) there can be some overlap $\backslash$ \\
\hline 34 & & $(0.5)$ \\
\hline 35 & US & so you can A:DD/ (0.5) to . a salty . dish (0.7) some sweet ingredients $\backslash$ \\
\hline 36 & & $(0.8)$ \\
\hline 37 & US & and in SOME other countries the two universes ... ARE .. fully separated $\backslash$ \\
\hline 38 & & $(0.5)$ \\
\hline 39 & US & so people DO not tend/ ... to mix/ (0.6) sweet . and salty/ .. euh:: items $\backslash$ food items $\backslash$. ok $\backslash$ \\
\hline 40 & & $(0.25)$ \\
\hline 41 & US & $\begin{array}{l}((\text { claquement de langue }))((\text { aspiration })) \text { and the la-\&the- ... the next you know } \backslash \text {. its local } \\
\text { taste for similar items/ O:R/. } \\
\text { different use } \backslash[. . .]\end{array}$ \\
\hline
\end{tabular}

US : l'enseignant, Ets : les étudiants

Dans l'ensemble de cet extrait, le point central de la présentation de l'enseignant consiste à exposer l'influence du local taste sur les patterns de consommation dans les différentes régions / cultures. Pour ce faire, il entame une présentation que l'on pourrait découper en trois phases: dans la première (1-16), US introduit le nouveau topic (local taste for similar items) et demande ensuite si l'explication n'a pas déjà été fournie (aucune réponse des étudiants), faisant allusion à l'objet süss versus sucré. Dans la deuxième phase (17-40), US présente deux exemples (l'un linguistique, l'autre culturel) liés au topic en reprenant les termes süss et sucré, en y ajoutant sweet, et en développant l'opposition entre sweet et salty. Il élabore ensuite le traitement de ces exemples et conclut dans la troisième phase (41).

Notre intérêt dans cette séquence est donc d'observer comment US développe son topic, quels objets il choisit en guise d'exemples et de quelle nature ils sont, ainsi que les différents modes de traitement auxquels il recourt, afin de comprendre si et 
comment le plurilinguisme est exploité en tant que ressource. Pour ce faire, revenons sur la deuxième phase de sa présentation.

En 17-18, US introduit les lexèmes süss, sucré et sweet: l'exemple qu'il choisit pour illustrer son topic «local taste for similar items » est donc plurilingue et thématise une possible différence entre ces lexèmes dans chacune des langues (il modalise pure lexical equivalents en recatégorisant par more or less). Après avoir introduit l'idée de " jugement positif ou négatif » sur sweet et süss (19), il introduit encore les notions de "sens figuré » et de «sens propre » qu'il applique au lexème anglais (my sweet darling) (20) pour ensuite démontrer la non-traductibilité du sens figuré de sweet par sucré en français dans une expression donnée. Il s'agit bien ici d'un mode de traitement métalinguistique sur un exemple plurilingue. Mais il faut noter que jusqu'ici, la dimension plurilingue est uniquement en mention, en citation, et que si elle est exploitée en tant qu'exemple, elle ne l'est pas en tant que moyen³.

Après une plaisanterie (24-26), US opère un véritable saut topical en introduisant two sort of taste (sweet et salty, 29): sweet n'est plus traité en opposition à ses équivalents français et allemand, mais en opposition à salty: le mode de traitement n'est plus métalinguistique. Dans la dernière partie de cette présentation, US présente un premier cas de figure (les overlaps qu'il peut exister entre sweet et salty (33-35), puis l'opposition des deux goûts (37-39). Sans transition (et sans laisser de place à d'éventuelles questions), US clôt ce topic et passe rapidement au suivant.

Le degré de plurilinguisme présent dans cet exemple est plutôt bas et se décline sous deux formes: dans des marques transcodiques (calques de L1 sur L2) et comme objet d'étude traité (ici sous forme de transferts), mais n'est pas exploité sous forme de codeswitching (dorénavant CS) dans l'organisation du discours.

81 Nous faisons l'hypothèse qu'un premier degré de plurilinguisme dans cet enseignement se manifeste par des traces de la L1 de l'enseignant sur son enseignement en L2. Le discours de US semble en effet exhiber un certain nombre de traces d'une pratique bilingue (autre que des phénomènes de transfert ou de CS) qui pourraient avoir un impact sur l'élaboration de savoirs. Premièrement, une partie des explications fournies par l'enseignant sont effectuées à l'aide de structures grammaticales simples et d'un lexique de base. Ainsi, les formulations et reformulations (cf. p. ex. 39) se caractérisent par une certaine simplification de contenus relativement complexes qui pourraient se révéler bénéfiques pour la médiation de et l'accès à ces derniers. Deuxièmement, il semble y avoir des phénomènes de calques de la L1 sur la L2 dans la syntaxe et l'organisation générale du discours (p. ex. l'introduction de topiques), selon les renseignements fournis par quelques locuteurs anglais $\mathrm{L}^{4}$. Cela pourrait avoir un certain impact au niveau de l'intercompréhension dans la mesure où ce calque pourrait faciliter la transmission des informations et la compréhension pour les étudiants francophones 5 . Une prochaine étape de notre recherche concernant cette pratique consistera donc à comparer les explications construites dans ce cours donné en L2 et celles produites par le même enseignant en L1 («cours-contrôle») ainsi qu'une recherche plus approfondie sur les éventuels calques grâce à l'aide d'informateurs anglophones.

82 Au-delà de ces marques transcodiques, cet extrait-ainsi que la pratique dont il provient - se caractérise par un degré de plurilinguisme relativement bas. Par exemple, le CS, vu comme une pratique exploitant deux (ou plusieurs) langues pour la communication et fonctionnant comme une ressource du répertoire bi-/ plurilingue, 
n'est pas une pratique courante dans le cours en question. D'autres langues interviennent principalement sous forme de transferts, c'est-à-dire d'unités isolées, insérées dans le discours en anglais et prises comme objets d'exemplification / d'explication. Dans ce cas, ces autres langues ne sont pas employées comme des moyens de communication, mais restent au niveau de la citation.

De ce fait, il n'y a, à première vue, pas d'exploitation de stratégies plurilingues comme ressources au service de l'interaction didactique dans la mesure où les interlocuteurs ne se servent pas explicitement de ressources bi-/ plurilingues, comme l'alternance codique. Le plurilinguisme ne semble pas tant exploité comme stratégie (communicative), mais la stratégie (didactique) consisterait à prendre le plurilinguisme comme objet.

Or dans l'entretien, lorsqu'il déclare que le plurilinguisme est une «ressource inexploitée ", il faut y voir un sens très restreint de la notion de ressource. Il s'agit avant tout de mettre l'accent sur les langues en tant qu'objets d'étude au service de la mise en œuvre du marketing (politiques concrètes), et moins sur le rôle des langues / du langage dans la construction conceptuelle et dans la transmission des savoirs dans sa discipline. Ceci pourrait être en lien direct avec quelques observations faites au niveau des séquences enregistrées, notamment la déconnexion entre la dimension linguistique (et culturelle) et la dimension disciplinaire. Caractère déconnecté relevé par l'enseignant lui-même concernant l'acquisition de la part des étudiants. Selon lui (et les résultats des examens), les étudiants arrivent mal à intégrer la dimension inter-linguistique et interculturelle dans leurs savoirs et savoir-faire en marketing. Les étudiants ne semblent pas seulement déstabilisés par la polysémie induite par le plurilinguisme (ils veulent une vérité univoque), mais pas non plus suffisamment formés ou "sophistiqués » (ou aware) pour des pratiques plurilingues. Une condition de réussite du plurilinguisme serait donc, selon l'enseignant, une sélection plus sévère des étudiants.

Le peu d'alternances codiques observées dans sa pratique et dès lors la non exploitation du plurilinguisme comme ressource ou comme stratégie communicative trouverait une explication dans ses déclarations. Et dans ce sens, sa pratique d'enseignement apparaît comme l'application directe de ses stratégies linguistiques déclarées. Fait remarquable si l'on considère nombre d'autres terrains où les pratiques effectives dépassent largement leurs possibilités d'explicitation, où les acteurs exploitent le facteur plurilingue à des fins plus variées que ce qu'ils pensent et disent faire, et montrant par là des décalages considérables entre pratiques et représentations. Ici, l'exploitation de la dimension plurilingue - telle que théorisée par l'enseignant pour sa matière - atteint de sérieuses limites dans la construction du discours didactique et, apparemment, dans la construction des savoirs par les étudiants. Comme si, en quelque sorte, ses représentations explicites et contrôlées l'enfermaient dans un schéma très rigide et l'empêchait de recourir à d'autres stratégies plus spontanées et variées, où le plurilinguisme serait exploité comme moyen et non seulement comme objet de réflexion.

\section{Remarques conclusives}

86 C'est ce que nous conviendrons d'appeler l'une des conditions majeures d'un impact possible et pertinent des politiques linguistiques sur les pratiques et dans ce sens que le 
projet Dylan s'inscrit dans l'optique d'une recherche "policy driven», impliquant un nouveau mode d'interaction avec une politique inscrite elle-même dans l'optique d'une politique "research-driven». Il s'agit d'un nouveau type de dialogue engagé dès le départ dans la formulation de la problématique elle-même et soutenu de façon constante dans le développement du processus de recherche, et impliquant des reformulations successives de la question de départ en fonction de l'évolution du contexte.

Nous parlerons ici d'une recherche en prise directe sur l'action, sur l'action politique et éducative notamment, d'une recherche « incarnée » ou « enacted », dans l'optique d'une " cognition située » ou d'une science engagée. Et à l'heure où les institutions de recherche sont interpellées sur l'utilité sociale et politique de la recherche, nous pensons déjà pouvoir apporter quelques éléments de réponse, ou tout au moins inviter responsables politiques et institutionnels et responsables scientifiques à envisager de nouveaux modes d'interaction et de nouvelles formes de synergies.

\section{BIBLIOGRAPHIE}

www.dylan-project.org

\section{NOTES}

1. Enseignement d'une Matière Intégrée à une Langue Étrangère

2. Citation tirée du semi-directif: "J'estime, quand un concept a été exposé en anglais à la base, pourquoi le le traduire, hein ?"

3. Nous observons ici des transferts mais pas de code-switching, selon la typologie de Auer (2000).

4. Cette hypothèse est également soutenue par une anecdote relatée par l'enseignant lors de l'entretien semi-directif. Ayant publié un ouvrage sur le marketing en français, il en a élaboré une version anglaise. Actuellement, il est en négociation avec l'éditeur anglophone qui lui demande notamment de défranciser le texte dans le sens de rendre plus anglophone la structure et d'"épurer" les formulations.

5. En revanche, le même phénomène pourrait se révéler un obstacle pour les non-francophones.

\section{RÉSUMÉS}

Le projet Dylan est un projet intégré (IP) du 6e programme-cadre européen, issu de la priorité 7 "Citoyenneté et gouvernance dans une société fondée sur la connaissance », rassemblant dixneuf universités partenaires de 12 pays européens. 
Le projet traite de la thématique 3.3.1. consistant à savoir si et comment une société européenne fondée sur la connaissance, visant à assurer le développement économique et la cohésion sociale, peut être créée en dépit d'une diversité linguistique plus importante suite à l'élargissement de l'Union Européenne. L'objectif essentiel du projet consiste à montrer sous quelles conditions la diversité linguistique qui prévaut en Europe constitue un atout plutôt qu'un obstacle.

Il vise en particulier à saisir en quoi la mise en œuvre de répertoires plurilingues contribue à la construction et au transfert des connaissances (atout cognitif) et interviennent dans le contrôle de la communication, la résolution de problèmes et la prise de décision (atout stratégique), dans la diversité des contextes économiques, politiques et éducatifs.

Composed of 19 universities from 12 countries, the Dylan Project is an integrated project (IP) of the $6^{\text {th }}$ Framework Programme of the European Union, under Priority 7 "Citizenship and governance in a knowledge-based society ».

The project addresses the core issue underlying topic 3.3.1: establish whether and how a European knowledge based society designed to ensure economic competitiveness and social cohesion can be created within a European Union that is linguistically more diverse than ever. The overarching objectives are to show that, in this respect, the linguistic diversity prevalent in Europe is potentially an asset rather than an obstacle and to identify the conditions under which individual and societal multilingualism can be turned to advantage.

It will show in what ways the usage of multilingual repertoires can promote the creation and the transfer of knowledge (cognitive asset) and have an impact on communication control, problem solving and decision making (strategic asset), in the diversity of economic, political and educational contexts.

\section{INDEX}

Keywords : language dynamics, linguistic diversity, cognitive asset, strategic asset

Mots-clés : dynamique des langues, gestion de la diversité, atout cognitif, atout stratégique

\section{AUTEUR}

\section{ANNE-CLAUDE BERTHOUD}

Anne-Claude Berthoud est professeure de linguistique à l'Université de Lausanne. Ses domaines d'enseignement portent essentiellement sur la linguistique de l'interaction et la linguistique de l'acquisition. Ses champs de recherche sont en étroite relation avec ses domaines d'enseignement, et en particulier la construction discursive et interactionnelle des objets de connaissances et les enjeux du plurilinguisme pour la construction et la circulation des savoirs (linguistiques et non linguistiques),thématiques-clés du Projet européen Dylan (Dynamiques des langues et gestion de la diversité) qu'elle dirige actuellement (2006 2011). Elle est en outre présidente de l'Académie suisse des sciences humaines et sociales, vice-présidente du Conseil européen pour les langues et vice-présidente du Fond National Suisse de la recherche scientifique Courriel : anne-claude.berthoud[at]unil.ch 